\title{
A Survey on Image Denoising Techniques
}

\author{
S.Preethi \\ Department of information technology \\ Karunya University \\ Coimbatore,India
}

\author{
D.Narmadha \\ Department of information technology \\ Karunya University \\ Coimbatore,India
}

\begin{abstract}
Image processing is an important charge in image denoising as a process and component in various other process There are many ways to denoise an image. The ultimate idea of this paper is to acquiesce better results in terms of quality and in removal of different noises. This paper is compared with three methods NL Means, NL-PCA, and DCT.PSNR and SSIM are used for quantitative study of denoising methods.
\end{abstract}

General Terms - Image denoising, Quality, Rician noise.

\section{INTRODUCTION}

Image denoising plays an essential role in medical image Most of the Medical images have low contrast objects corrupted by random noise in the input process. During transmission and retrivel of an image ther is more possibility of corruption.Denoising is basic task required by medical analysis.Noise removal causes blurring of images,the quality of image is also lowered.Nonlinear models can hold edges in an enhanced mode than linear models. The main properties of a good image denoising model is that it will remove noise while preserving edges. conventionally, linear models have been used. One common approach is to use filters. For some purposes this kind of denoising is satisfactory. One big advantage of linear noise removal models is the speed. But drawback of the linear models is that they are not able to preserve edges in a well manner. Edges, which are recognized as discontinuities in the image, are tarnished out. Nonlinear models on the other hand can handle edges in a better way than linear models. Nonlinear filter is a signalprocessing device where its output is not a linear function of its input. Competence of this paper can be experienced on both real and simulated medical images.Rician noise utmost occurs in MRI.It adds more problems in low SNR.

\section{METHODOLOGY RELATED TO IMAGE DENOISING}

\subsection{Two Stage Image Denoising By Principal Component Analysis With Local Pixel Grouping}

In this paper there are two stages. In First stage noisy image is taken as an input and subjected to local pixel grouping and then to principal component transform where, they convert set of observed correlated values to linearly uncorrelated variables.

Number of PC is equal or less than the original values. Then passed to inverse PCA transform and denoised output is taken as a result.

A new algorithm called BM3D is developed, where block matching is done and matched blocks from frames are grouped together on the basis of similarity.Fig. 2 details the stages of image denoising by PCA with LPG. Average weight of all overlapping block is anticipated.

Pro of this paper is it preserves local image structures while denoising,Con is due to low peak signal to noise ratio the quality of image is low.

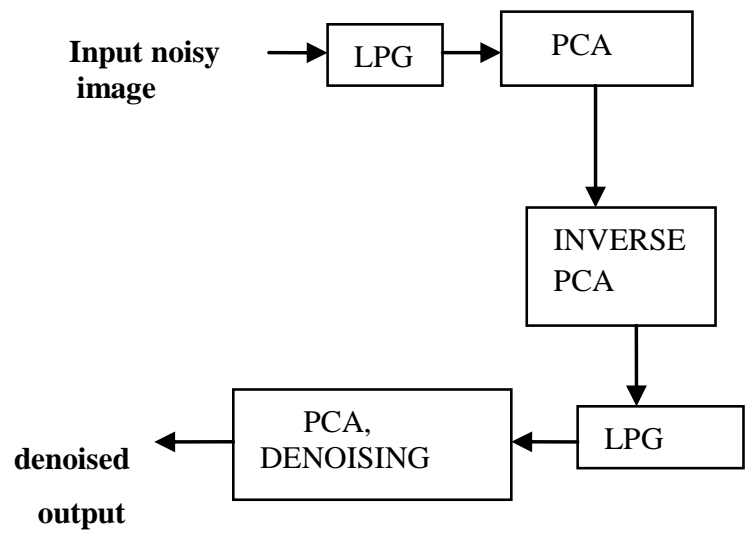

Fig 1: Flowchart of two stage scheme

\subsection{K-SVD An Algorithm For Designing Overcomplete Dictionaries For Sparse Representation}

MOD and MAP methods are involved in this paper KSVD.MOD(method of direction )this method is similar to $\mathrm{k}$ means clustering. $\mathrm{N}$ observations are partitioned to $\mathrm{k}$ clusters and each observations belongs to each cluster by its nearest mean value.MAP method is used for increasing the closeness to single value detection. It combines with MOD efficience and recovers dictionary. PSNR is high for compression. It requires some more methods for solving problem with large image patches. Main goal is to find D that yields sparse representation for training signal.

Updating of one column of $\mathrm{D}$ at a time is problem having straight solution on SVD.while updation coefficient value change. Pro is it fills the missing pixels in an image.

\subsection{Performance Analysis For Non Linear Filering Algorithm For Underwater Images}

They use 5 algorithms for removing 3types of noises:

- Speckle noise

- Salt and Pepper noise

- Gaussian noise 
In median filter original and result pixel filter has same pixels In component median filter, it is applied over point in a signal. In Vector median filter, mask is applied over one point. In spatial median filter removal of noise is similar to vector median filter. Modified SMF, replaces every point, which is useful for different noise removals.

Median filter removes noise in input with large magnitude. In Modified SMF every point is replaced by smoothing. Modified SMF is useful for different noise removal.

Pros are Component median filter is more accurate than median filter.Cons are Component median creates a new signal point that doesnot exits in original signal and Uncorrupted datas are also removed unknowingly.

\subsection{Curvelet Transform For Image Denoising}

Here, ridgelet alone cannot provide capable work. So curvelet along ridgelet methods are used.Localize the ridgelets, Edges are always discontinuities where curvelets give exact curve like appearance but ridge lets are straight rather than a curve.Curvelets based on multiscale ridgelets show sparsity in representation of smooth edges. High perceptual quality. Software for computing is still in decisive stage. Discrete curvelet rigelet shows spasity in representation of smooth and fine edges.Input is taken as a digital input on catesian grid by considering the problem like white noise apply threshold in curvelet to produce denoised output.Pro is Curvelets obtains small MSE of reconstruction than wavelets.Con is Software for computing is in formative stage.

\subsection{Multiresolution Denoising For Optical} Coherence Tomography

In OCT, optical signal is taken as an input and processed using long wavelength light penetrates to scattering medium. Different fifteen optical coherence tomography images are extracted and tested with different filters for checking whether they are visually effective.Compared to single resoultion, multiresolution filters improves the quality of OCT images. Tested with two single resolution and four multiresolution filters where, single resolution filters were inferior to multiresolution filters. Wavelet and gamma expression priors(WGE) preserves edges and textures but uniform areas are also less flattened.

Pro is it Provides high resolution than the MRI, Ultrasound etc. Feature based threshold doesnot preserves edges. Con is FBT doesnot preserves edges and textures.

\subsection{Non Local Means Denoising For Medical Images}

Based on rician model iterative NL-Means and other method NL-PCA is compared. In this paper the proposed method has distinctive features like firstly, usage of restricted local neighbourhood secondly, weight calculation, finally applying kernel.

Fig.1 describes the input as scanned medical image and by applying NL Means filter rician noise is removed and denoised image is taken as an resulted output.

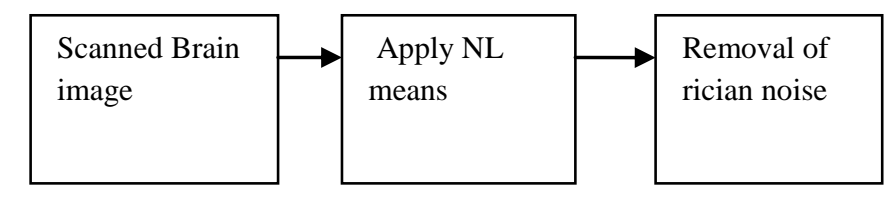

Fig 2: NL Means Sample Input Output

PSNR(peak signal to noise ratio) value is high and they use another similarity measure named SSIM(structural similarity index matrix) It measures the similarity between two image value ranges between 0 and 1 .

PSNR and MSE are inconstant with human eye perception so they calculated SSIM.

\section{ANALYSIS OF DIFFERENT ALGORITHM AND TECHNIQUES RELATED TO DENOISING}

The main parameters considered for analysis of denoising process is Peak signal to noise ratio,structural similarity index matrix and quality of an image.Quality of an image always has an important concerns in image denoising process.Many new algorithms and implementation has been presented for improving the quality of an image.

\subsection{Merit Of Two Stage Image Denoising By Principal Component Analysis With Local Pixel Grouping \\ i) Preserves local image structure during denoising.}

\subsubsection{Demerits Of Two Stage Image Denoising}

By Principal Component Analysis With Local

Pixel Grouping

i) PSNR is low.

ii)Quality of image is not good.

\subsection{Merit Of K-SVD An Algorithm For Designing Overcomplete Dictionaries For Sparse Representation}

i) Fills the missing pixels in an image and good in compression.

i) It requires some more methods for solving problem with large image patches.

\subsubsection{Demerit of K-Svd An Algorithm For Designing Overcomplete Dictionaries For} Sparse Representation

i) It requires some more methods for solving problem with large image patches.

\subsection{Merits Of Performance Analysis For Non Linear Filering Algorithm For Underwater Images}

i) Component median filter is more accurate than median filter.

ii) Modified SMF is useful for different noise removal. 
3.3.1 Demerits Of Performance Analysis For Non Linear Filtering Algorithm For Underwater Images

i) Component median creates a new signal point that doesnot exits in original signal.

ii)Uncorrupted datas are also removed unknowingly.

\subsection{Merit Of Curvelet Transform For}

\section{Image Denoising}

i) Curvelets obtains small MSE of reconstruction than wavelets.

\subsubsection{Demerit Of Curvelet Transform For}

Image Denoising

i) Software for computing is in developing stage.

\subsection{Merit Of Multiresolution Denoising For Optical coherence Tomography}

i) Provides high resolution than the MRI,Ultrasound etc.

\subsubsection{Demerits Of Multiresolution Denoising For} Optical coherence Tomography

i) Feature based threshold doesnot preserves edges.

ii) WGE preserves edges but uniform areas are also less flattened.

\subsection{Merits Of Non Local Means Denoising For Medical Images}

i) $2 \mathrm{D}$ images corrupted by additive white Gaussian noise are removed.

ii) Quality of image is comparatively high.

\subsubsection{Demerits Of Non Local Means Denoising For Medical Images \\ i) Use of global noise variance leads to suboptimal results. \\ ii) Reduces the visibility of certain features.}

\section{EXPERIMENTAL EVALUATION}

Recently proposed methods were compared with proposed algorithm for evaluating the results

i) NL- PCA: "Non local principal component analysis method", neighborhood size was $3 * 3 * 3$ during experiments.

ii) NL-Means:"Non local means denoising", neighborhood size was $3 * 3 * 3$ and search window size was $9 * 9 * 9$ during experiments.

Based on similarity measure they were compared and PSNR value was comparatively high in the proposed algorithm. Figure 4 expains the quantitative analysis of other method, NL-Means with the proposed method based on similarity measure like PSNR and SSIM.Background was removed for qualitative analysis.It can be noticed from the graph that the proposed method performance is better for similarity measure.
1

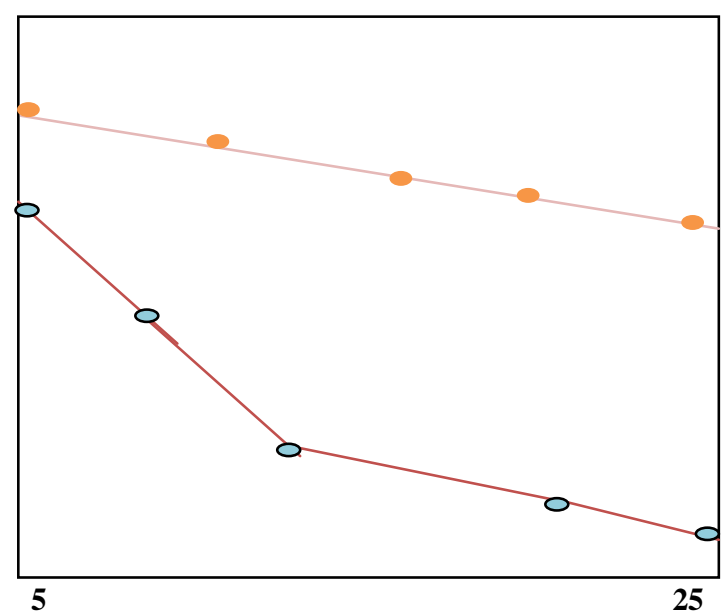

Fig 4: Analysis of proposed method with NL-Means based on PSNR,SSIM

$$
\begin{aligned}
& \text { NL-Means } \\
& \text { o Proposed method }
\end{aligned}
$$

Table1,2,3,4,5.compares the other methods with the parameters such as technique concerned and efficiency measure. Here, efficiency is evaluated by the terms of PSNR,SSIM,SNR.

Table1.Comparision of Proposed paper with Reference1

\begin{tabular}{|l|c|c|}
\hline Parameters & Proposed paper & Ref paper1 \\
\hline Technique & $\begin{array}{c}\text { Iterative NL-Means } \\
\text { algorithm removes } \\
\text { noise from 2d } \\
\text { Images corrupted by } \\
\text { additive white } \\
\text { gaussian noise }\end{array}$ & $\begin{array}{c}\text { Noisy image is } \\
\text { sent to to local } \\
\text { pixel grouping } \\
\text { and by pca } \\
\text { transform and } \\
\text { inverse PCA } \\
\text { denoised output } \\
\text { obtained, } \\
\text { BM3D algorithm } \\
\text { developed. }\end{array}$ \\
\hline Efficiency & $\begin{array}{c}\text { PSNR value is high, } \\
\text { Measure }\end{array}$ & $\begin{array}{c}\text { PSNR value is } \\
\text { low, } \\
\text { SSIM value is } \\
\text { high. }\end{array}$ \\
\hline
\end{tabular}

Table2.Comparision of Proposed paper with Reference2

\begin{tabular}{|l|c|c|}
\hline Parameters & Proposed paper & Ref paper2 \\
\hline employned & $\begin{array}{c}\text { Iterative NL-Means } \\
\text { algorithm removes } \\
\text { noise from 2d Images } \\
\text { corrupted by additive } \\
\text { white gaussian noise }\end{array}$ & $\begin{array}{c}\text { MOD works similar } \\
\text { to k means } \\
\text { clustering and } \\
\text { MAP method } \\
\text { increases closeness } \\
\text { to }\end{array}$ \\
Efficiency & $\begin{array}{c}\text { PSNR value is high, } \\
\text { MSIM value is high. }\end{array}$ & $\begin{array}{c}\text { Comparing to the } \\
\text { proposed paper } \\
\text { PSNR value is low. }\end{array}$ \\
\hline
\end{tabular}


Table3.Comparision of Proposed paper with Reference3

\begin{tabular}{|c|c|c|}
\hline Parameters & $\begin{array}{l}\text { Proposed } \\
\text { paper }\end{array}$ & Ref paper3 \\
\hline Technique employed & $\begin{array}{l}\text { Iterative NL- } \\
\text { Means } \\
\text { algorithm } \\
\text { removes noise } \\
\text { from } 2 \mathrm{~d} \text { Images } \\
\text { corrupted by } \\
\text { additive white } \\
\text { gaussian noise }\end{array}$ & $\begin{array}{l}\text { Three types } \\
\text { of noises are } \\
\text { removed by } \\
\text { applying five } \\
\text { algorithms. } \\
\text { Modified } \\
\text { SMF is } \\
\text { useful for } \\
\text { different } \\
\text { noise } \\
\text { removal. }\end{array}$ \\
\hline Efficiency Measure & $\begin{array}{l}\text { PSNR value is } \\
\text { high, } \\
\text { SSIM value is } \\
\text { high. }\end{array}$ & $\begin{array}{l}\text { PSNR is } \\
\text { high in } \\
\text { MSMF } \\
\text { filter,MSE is } \\
\text { low in } \\
\text { median } \\
\text { filter. }\end{array}$ \\
\hline
\end{tabular}

Table4.Comparision of Proposed paper with Reference4

\begin{tabular}{|l|c|c|}
\hline Parameters & Proposed paper & Ref paper4 \\
\hline Technique & $\begin{array}{c}\text { Iterative NL- } \\
\text { employed } \\
\text { removes noise } \\
\text { from 2d Images } \\
\text { corrupted by } \\
\text { additive white } \\
\text { gaussian noise }\end{array}$ & $\begin{array}{c}\text { Curvelet with } \\
\text { ridgelet used to } \\
\text { provide efficient } \\
\text { work on digital } \\
\text { inputs and denoised } \\
\text { output. Curves are } \\
\text { recovered sharply. }\end{array}$ \\
\hline $\begin{array}{l}\text { Efficiency } \\
\text { Measure }\end{array}$ & $\begin{array}{c}\text { PSNR value is } \\
\text { high, } \\
\text { SSIM value is } \\
\text { high. }\end{array}$ & $\begin{array}{c}\text { PSNR value is } \\
\text { better in multiscale } \\
\text { entropy with some } \\
\text { few noise. }\end{array}$ \\
\hline
\end{tabular}

Table5.Comparision of Proposed paper with Reference5

\begin{tabular}{|l|c|c|}
\hline Parameters & Proposed paper & Ref paper5 \\
\hline Technique & $\begin{array}{c}\text { Iterative NL- } \\
\text { Means algorithm } \\
\text { removes noise } \\
\text { from 2d Images } \\
\text { corrupted by } \\
\text { additive white } \\
\text { gaussian noise }\end{array}$ & $\begin{array}{c}\text { Different OCT } \\
\text { images are } \\
\text { extracted and } \\
\text { tested with } \\
\text { various filters } \\
\text { for checking } \\
\text { visual } \\
\text { effectiveness }\end{array}$ \\
\hline Efficiency & $\begin{array}{c}\text { PSNR value is } \\
\text { high, } \\
\text { Measure }\end{array}$ & $\begin{array}{c}\text { PSNR is low for } \\
\text { FBT compared } \\
\text { to }\end{array}$ \\
& hSIM value is & WGE. \\
\hline
\end{tabular}

\section{CONCLUSION}

NL- Means is the new method proposed to denoise images mainly of medical images. Similarity measures like PSNR, SSIM, SNR was compared with other recently proposed
methods.A literature survey for various image denoising process was done.Proposed method can provide better results in terms of image quality and similarity measures.Future scope is to calculate the amount of noise added to the pixel, removal of noise and evaluating the signal to noise ratio.

\section{ACKNOWLEDGMENTS}

The authors would like to thank ALMIGHTY GOD whose blessings have bestowed in me the will power and confidence to carry out my work

\section{REFERENCES}

[1] L.Zhang, W. Dong, D. Zhang, and G. Shi, "Two-stage image denoising by principal analysis with local pixelgrouping," Pattern Recognition, vol. 43, no. 4, pp. 1531-1549,2010.

[2] M. Aharon, M. Elad, and A. Bruckstein, "K-SVD: an algorithm for designing overcomple dictionaries for sparse representation,'IEEE Transactions on Signal Processing, vol. 54, no.11,pp. 4311-4322, 2006.

[3] M. Elad, M. Aharon, "Performance and analysis for non linear filtering algorithm for under water images"International Journal of Computer Science and Information Security, vol.6, no2,pp., 2009.

[4] A. Pizurica and W. Philips, "Curvelet transform for image denoising," IEEE Transactions on Image Processing, vol. 11, no. 6, pp. 654-665, 2002.

[5] M. Elad and M. Aharon, "Multiresolution denoising for optical coherence tomography," IEEE Transactions on Image Processing, vol. 4, 2008

[6] Ke Lu,Ning $\mathrm{He}$ and LiangLi,"Non local Means denoising for medical images",Computational and Mathematical Methods in Medicine,volume2012.

[7] M. Elad and M. Aharon, "Multiresolution denoising for optical coherence tomography," IEEE Transactions on Image Processing, vol. 4, 2008.

[8] Dewey tucker, Hamid Krim and David Donoho, "On denoising and best signal representation" IEEE Transactions on information Theory,vol.45.no.7.1999.

[9] G. Y. Chen and B. K'egl, "Image denoising with complex ridgelets,"Pattern Recognition, vol. 40, no. 2 , pp. 578-585, 2007.

[10] M. Elad and M. Aharon, "Image denoising via sparse and redundant representations over learned dictionaries," IEEE Transactions on Image Processing,vol 15,no.12,pp.3736-3745,2006.

[11] R. Gastaud and J. L. Starck, "Dynamic range compression : A new method based on wavelet transform," in Astron. Data Anal. Software Systems Conf., Strasbourg, 2003.

[12] J. L. Starck, M. Elad, and D. L. Donoho, "Image decomposition: Separation of texture from piece-wise smooth content," in SPIE Conf. Signal Image Process. Wavelet Application. Signal Image Process. X, SPIE $48^{\text {th }}$ Annu. Meeting, San Diego, CA, Aug. 3-8, 2003. 